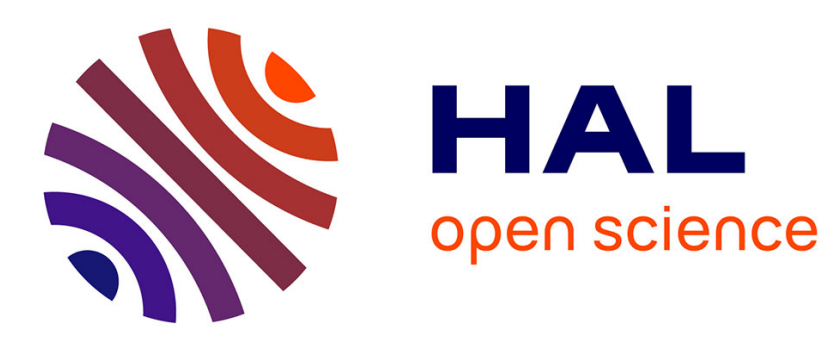

\title{
Anti-windup strategy for reset control systems
}

\author{
Sophie Tarbouriech, Thomas Loquen, Christophe Prieur
}

\section{To cite this version:}

Sophie Tarbouriech, Thomas Loquen, Christophe Prieur. Anti-windup strategy for reset control systems. International Journal of Robust and Nonlinear Control, 2011, 20 (10), pp.1159-1177. 10.1002/rnc.1747. hal-00595218

\section{HAL Id: hal-00595218 \\ https://hal.science/hal-00595218}

Submitted on 24 May 2011

HAL is a multi-disciplinary open access archive for the deposit and dissemination of scientific research documents, whether they are published or not. The documents may come from teaching and research institutions in France or abroad, or from public or private research centers.
L'archive ouverte pluridisciplinaire HAL, est destinée au dépôt et à la diffusion de documents scientifiques de niveau recherche, publiés ou non, émanant des établissements d'enseignement et de recherche français ou étrangers, des laboratoires publics ou privés. 


\title{
Anti-windup strategy for reset control systems*
}

\author{
Sophie Tarbouriech ${ }^{a}$, Thomas Loquen $^{b}$, Christophe Prieur $^{c}$ \\ a. CNRS; LAAS; 7 avenue du Colonel Roche F-31077, Toulouse, France. \\ Université de Toulouse; UPS, INSA, INP, ISAE; LAAS; F-31077, Toulouse, France. tarbour@laas.fr \\ b. ONERA, System Control and Flight Dynamics Department, 2 avenue Edouard Belin, F-31055 Toulouse Cedex \\ 4, France. Thomas.Loquen@onera.fr \\ c. Department of Automatic Control, Gipsa-lab, Domaine universitaire, 961 rue de la Houille Blanche, BP 46, \\ 38402 Grenoble Cedex, France. christophe.prieur@gipsa-lab.grenoble-inp.fr.
}

\begin{abstract}
This paper proposes an anti-windup strategy to deal with stability and performance requirements for a class of hybrid systems, such as those including a reset controller and subject to input saturation. The computation of the anti-windup compensator aiming at ensuring both $\mathcal{L}_{2}$ input-to-state stability and internal stability of the closed-loop system is carried out from the solution of matrix inequalities. Depending on the way chosen to describe reset rules, the conditions for designing the anti-windup compensator are expressed through nonlinear matrix inequalities or linear matrix inequalities. Some optimization criteria in both cases are considered for the synthesis purpose: maximization of the $\mathcal{L}_{2}$-norm upper bound on the admissible disturbances for which the trajectories are assured to be bounded; minimization of the $\mathcal{L}_{2}$-gain of the disturbance to the system to-be-controlled output; and the maximization of an estimate of the domain of attraction of the origin.
\end{abstract}

Keywords: Reset control systems, saturation, stability regions, anti-windup technique

\section{Introduction}

The anti-windup strategy is a well-known and efficient technique to cope with undesirable effects (on both performance and stability) induced by actuator saturation in control loops. The design of antiwindup compensators were first motivated by dealing with the degradation of the transient performance induced by saturation in feedback control systems containing integral actions [10]. More recently, the anti-windup compensators design through formal and systematic methods has emerged (see, for example, $[9,15,18,27,29]$, for a large recent overview). In particular, these works present approaches based on LMI conditions (or quasi-LMI, i.e. non-convex condition but with simple nonlinearity). The advantage of the LMI-based methods lies on the fact that the anti-windup design can be carried out through convex optimization problems, allowing to address different optimal synthesis criteria (such as $\mathcal{L}_{2}$-gain attenuation or enlargement of the basin of attraction) in an optimal way.

Moreover, in the quest for providing more flexible stabilizing tools, research efforts have focused on developing control algorithms, and using controllers that involve switching, or on-line adaptation. Adding some hybrid loops may be fruitful in comparison to linear controllers. See e.g. $[1,8]$ for some academic examples, and consider $[4,5,16,34]$ for specific control applications where hybrid controllers are used. Even if the analysis of the stability may become complicated, the performance improvement of hybrid strategies with respect to more classical controllers (i.e. regular linear or nonlinear controllers) is now proved (see, e.g., [6, 21, 22, 24, 11, 32], for different use of hybrid controllers). However, such a class of

\footnotetext{
${ }^{*}$ Work supported in part by the ANR project ArHyCo, ARPEGE, contract number ANR-2008 SEGI 004 01-30011459.
} 
feedback laws may introduce - possibly unrealistic - large transition input values. Indeed, many physical control systems are subject to magnitude-limitation in the input. A first work studying the stability issue of a nonlinear systems resulting from a FORE and a saturation in the input is proposed in [20].

In this paper, a SISO linear system controlled by a reset controller and subject to saturation is considered. The considered hybrid controller is that one that combines a continuous linear-based dynamic (represented by differential equations) with a reset dynamic (instantaneous jumps to zero of variables). Continuous trajectories are active in a subset of the state space, called the flow set. Resets of whole or part of system states correspond to an instantaneous jump and are only active in the subset of the state space, called jump set. The current paper proposes some extensions of previous works (in particular [1, 21]) to synthesize a dynamic anti-windup compensator. This paper proposes in particular an anti-windup strategy to deal with stability and performance requirements for a class of hybrid systems, such as those including a reset controller and subject to input saturation. At this aim, different types of reset rule and therefore different flow and jump sets are considered. In both cases, constructive conditions leading to quasi-LMIs or LMIs, based on the use of suitable Lyapunov functions, modified sector condition and the application of adequate congruence transformations, are proposed to compute anti-windup compensators. The computation of the anti-windup compensator aiming at ensuring both $\mathcal{L}_{2}$ input-to-state stability and internal stability of the closed-loop system is then carried out from the solution of convex optimization problems, including the maximization of the $\mathcal{L}_{2}$-norm upper bound on the admissible disturbances for which the trajectories are bounded; the minimization of the $\mathcal{L}_{2}$-gain of the disturbance to the system regulated output; and the maximization of an estimate of the domain of attraction of the origin.

This paper is organized as follows. The problem to be treated is formally stated in Section 2. In Section 3 , theoretical results on the anti-windup synthesis are developed. Two reset conditions are considered. Section 4 addresses the computational and numerical issues regarding the theoretical conditions previously developed. Convex optimization schemes and an illustrative example are also presented. Finally, Section 5 contains some concluding remarks and presents forthcoming issues.

Notation. For two vectors $x, y$ of $\mathbb{R}^{n}$, the notation $x \succeq y$ means that $x_{(i)}-y_{(i)} \geq 0, i=1, \ldots, n$. For any vector $x \in \mathbb{R}^{n},|x|$ is its absolute value (i.e., $|x|_{(i)}=\left|x_{(i)}\right|, i=1, \ldots, m$ ) and $\|x\|$ is its Euclidian norm. The identity matrix of order $n$ is denoted $I_{n}$. The null $m \times n$ matrix is denoted $0_{m \times n}$. When no confusion is possible, identity and null matrices are simply denoted $I$ and 0 , respectively. For two symmetric matrices, $A$ and $B, A>B$ means that $A-B$ is positive definite. $A^{\prime}$ denotes the transpose of $A$. $\operatorname{diag}(A, B)$ denotes the diagonal matrix obtained from matrices $A$ and $B$. Furthermore, in the case of partitioned symmetric matrices, the symbol $\star$ denotes symmetric blocks. The convex hull defined by the vertices $v_{i} \in \mathbb{R}^{n}$ are denoted $C o\left\{v_{i}, i=1, \ldots, r\right\}$.

\section{Problem statement}

Consider the following SISO plant:

$$
\begin{aligned}
\dot{x}_{p} & =A_{p} x_{p}+B_{p} u+B_{w} w \\
y & =C_{p} x_{p} \\
z & =C_{z} x_{p}+D_{z} u
\end{aligned}
$$

where $x_{p} \in \mathbb{R}^{n_{p}}$ is the state vector, $y \in \mathbb{R}$ is the measured output of the plant, $u \in \mathbb{R}$ is the input of the plant, $w \in \mathbb{R}^{n_{w}}$ is the disturbance vector and $z \in \mathbb{R}^{n_{z}}$ is the regulated output for performance purpose. In (1), $A_{p}, B_{p}, C_{p}, C_{z}$ and $D_{z}$ are constant matrices of appropriate dimensions. Pairs $\left(A_{p}, B_{p}\right)$ and $\left(C_{p}, A_{p}\right)$ are assumed to be controllable and observable, respectively. Furthermore, the disturbance vector $w$ is assumed to be limited in energy, i.e., given a finite positive scalar $\delta$ :

$$
\|w\|_{2}^{2}=\int_{0}^{\infty} w(\tau)^{\prime} w(\tau) d \tau \leq \frac{1}{\delta}
$$


The input of the plant is supposed to be bounded in magnitude by $u_{0}>0$, i.e., $-u_{0(i)} \leq u_{(i)} \leq u_{0(i)}$, $\forall i=1, \ldots, m$. In consequence, the control signal to be effectively injected in the system considering the controller output $y_{c}$ is

$$
u=\operatorname{sat}\left(y_{c}\right)
$$

The saturation map is the classical function defined as $\operatorname{sat}\left(y_{c}\right)=\operatorname{sign}\left(y_{c}\right) \min \left(\left|y_{c}\right|, u_{0}\right)$, where $u_{0}>0$ denotes the level of saturation.

Associated to system (1), we consider a reset controller that leads to mixed discrete/continuous dynamics. This kind of systems is in the class of hybrid systems. Hence, by using the hybrid framework introduced in [12], the reset controller is described by:

$$
\begin{aligned}
& \left.\begin{array}{l}
\dot{x}_{c}=A_{c} x_{c}+B_{c} y \\
y_{c}=C_{c} x_{c}+D_{c} y \\
\dot{\tau}=1 \\
x_{c}^{+}=0 \\
\tau^{+}=0
\end{array}\right\} \text { if }\left(x_{p}, x_{c}\right) \in \mathcal{F}_{0} \text { or } \tau \leq \rho \\
& \text {. } \left.x_{c}\right) \in \mathcal{J}_{0} \text { and } \tau \geq \rho
\end{aligned}
$$

where $x_{c} \in \mathbb{R}^{n_{c}}$ is the state of the controller, $x_{c}$ is the state value before a jump and $x_{c}^{+}$after a jump. In (4), $A_{c}, B_{c}, C_{c}, D_{c}$ are constant matrices of appropriate dimensions, and constitute the so-called linear-based data of the hybrid controller. The variables $y$ and $y_{c}$ are the controller input and output, respectively. $\mathcal{F}_{0}$ and $\mathcal{J}_{0}$ correspond to the flow and jump sets. The variable $\tau$ corresponds to the so-called "temporal regularization". It allows to guarantee a lower bound of the time in between resets, called $\rho$. The objective to add this positive scalar $\rho$ is to avoid an infinite number of switches, i.e., to avoid Zeno solutions [12].

It is supposed that the controller (4) was computed to stabilize system (1), without taking into account the saturation of the input of the plant: $u=y_{c}$. In order to cope with such a disregarding, an antiwindup loop is then considered to minimize the effect of the saturation on the closed-loop performance and stability. At this aim, the following anti-windup compensator is considered:

$$
\begin{aligned}
& \dot{x}_{a}=A_{a} x_{a}+B_{a}\left(\operatorname{sat}\left(y_{c}\right)-y_{c}\right) \\
& y_{a}=C_{a} x_{a}+D_{a}\left(\operatorname{sat}\left(y_{c}\right)-y_{c}\right)
\end{aligned}
$$

leading to the modified controller:

$$
\begin{aligned}
& \left.\begin{array}{l}
\dot{x}_{c}=A_{c} x_{c}+B_{c} y+y_{a} \\
y_{c}=C_{c} x_{c}+D_{c} y \\
\dot{\tau}=1 \\
x_{c}^{+}=0 \\
\tau^{+}=0
\end{array}\right\} \text { if }\left(x_{p}, x_{c}, x_{a}\right) \in \mathcal{F} \text { or } \tau \leq \rho \\
&
\end{aligned}
$$

where $x_{a} \in \mathbb{R}^{n_{a}},\left(\operatorname{sat}\left(y_{c}\right)-y_{c}\right) \in \mathbb{R}, y_{a} \in \mathbb{R}^{n_{c}}$ are the state, the input and the output vectors of the anti-windup compensator. We recall some basic ingredients on hybrid system theory, and the notion of solutions of (1) in closed loop with (5) and (6) (see [12, 23, 22] and references therein). Due to mixed discrete/continuous dynamics, a solution of (1), (5) and (6) will be defined on a mixed discrete/continuous time domain. More precisely:

Definition $1 A$ set $\mathcal{D}$ is a hybrid time domain if for all $(T, J) \in \mathcal{D}, \mathcal{D} \cap([0, T] \times\{0,1, \ldots J\})$ is a compact hybrid time domain, i.e. it can be written as

$$
\bigcup_{j=0}^{J-1}\left(\left[t_{j}, t_{j+1}\right], j\right)
$$


for some finite sequence of times $0=t_{0} \leq t_{1} \ldots \leq t_{J}$. A solution to (1), (5) and (6) consists of a hybrid time domain $\mathcal{D}$ and functions $\left(x_{p}, x_{c}, x_{a}, \tau\right): \mathcal{D} \rightarrow \mathbb{R}^{n_{p}} \times \mathbb{R}^{n_{c}} \times \mathbb{R}^{n_{a}} \times \mathbb{R}$ such that $\left(x_{p}, x_{c}, x_{a}, \tau\right)(t, j)$ is absolutely continuous in $t$ for a fixed $j$ and $(t, j) \in \mathcal{D}$ satisfying

(S1) for all $j \in \mathbb{N}$ and almost all $t$ such that $(t, j) \in \mathcal{D}$, we have

$$
\left(x_{p}, x_{c}, x_{a}\right)(t, j) \in \mathcal{F} \text { or } \tau(t, j) \leq \rho
$$

and

$$
\begin{aligned}
\dot{x}_{p}(t, j) & =A_{p} x_{p}(t, j)+B_{p} u(t, j)+B_{w} w(t) \\
\dot{x}_{c}(t, j) & =A_{c} x_{c}(t, j)+B_{c} y(t, j)+y_{a}(t, j) \\
\dot{x}_{a}(t, j) & =A_{a} x_{a}(t, j)+B_{a}\left(\operatorname{sat}\left(y_{c}(t, j)\right)-y_{c}(t, j)\right) \\
\dot{\tau}(t, j) & =1 .
\end{aligned}
$$

(S2) For all $(t, j) \in \mathcal{D}$ such that $(t, j+1) \in \mathcal{D}$, we have

$$
\left(x_{p}, x_{c}, x_{a}\right)(t, j) \in \mathcal{J} \text { and } \tau(t, j) \geq \rho,
$$

and

$$
\begin{aligned}
x_{p}(t, j+1) & =x_{p}(t, j) \\
x_{c}(t, j+1) & =0 \\
x_{a}(t, j+1) & =x_{a}(t, j) \\
\tau(t, j+1) & =0 .
\end{aligned}
$$

This definition implies in particular that the state solution $\left(x_{p}, x_{c}, x_{a}, \tau\right)$ is parameterized by $(t, j)$ where $t$ is the ordinary time and $j$ is an independent variable that corresponds to the number of jumps of the solution. When the state belongs to the intersection of the flow set and of the jump set, then the solution can either flow or jump. Throughout the paper, we will always use the notation $\dot{x}_{p}$ for $\dot{x}_{p}(t, j)$ and $x_{p}^{+}$ for $x_{p}\left(t_{j+1}, j+1\right)$ (and similarly for the other components of the state).

This paper is devoted to the following problem.

Problem 1 Given flow and jump sets $\mathcal{F}$ and $\mathcal{J}$.

1. When $w=0$, determine the anti-windup compensator, i.e., matrices $A_{a}, B_{a}, C_{a}$ and $D_{a}$, and a region $\mathcal{E}$, as large as possible, in which the asymptotic stability of the closed-loop system (1), (5) and $(6)$ is guaranteed, i.e., $\mathcal{E}$ is included in the basin of attraction of the origin of closed-loop system.

2. When $w \neq 0$, the $\mathcal{L}_{2}$-gain from $w$ to $z$, in the case of null initial condition, for all disturbances $w$ satisfying (2) is such that: $\|z\|_{2}^{2} \leq \gamma\|w\|_{2}^{2}$.

The implicit objective in the first item of Problem 1 is to maximize the size of the basin of attraction for the closed-loop system (1), (5) and (6) (with $w=0$ ) over the choice of matrices $A_{a}, B_{a}, C_{a}$ and $D_{a}$. Such an objective can be accomplished indirectly by searching for an anti-windup compensator defined from $A_{a}, B_{a}, C_{a}$ and $D_{a}$ that leads to a region of stability $\mathcal{E}$ for the closed-loop system as large as possible, in particular in the $x_{p}$ or $\left(x_{p}, x_{c}\right)$ directions. On the other hand, when the open-loop system is asymptotically stable, it can be possible to search for the controller matrices in order to ensure the global asymptotic stability of the origin of the closed-loop system.

Moreover, Problem 1 will be addressed by considering different types of reset rule and therefore different flow and jump sets. Hence, we will study what is the influence of the reset rule in the solution of Problem 1. 


\section{Theoretical anti-windup design conditions}

\subsection{Preliminary results}

Let us define the augmented state vector

$$
x=\left[\begin{array}{l}
x_{p} \\
x_{c} \\
x_{a}
\end{array}\right] \in \mathbb{R}^{n}
$$

with $n=n_{p}+n_{c}+n_{a}$ and the following deadzone nonlinearity:

$$
\phi(K x)=\operatorname{sat}(K x)-K x, K=\left[\begin{array}{ll}
D_{c} C_{p} & C_{c} \mid 0
\end{array}\right]=\left[\begin{array}{l|l}
K_{1} \mid 0
\end{array}\right] \in \mathbb{R}^{1 \times n}
$$

Hence, by combining (1), (5), (6), (7) and (8), the closed-loop system reads:

$$
\left.\begin{array}{l}
\dot{x}=A_{F} x+B_{F} \phi(K x)+B_{2} w \\
y_{c}=K x \\
y=C x \\
z=C_{2} x+D_{z} \phi(K x) \\
\dot{\tau}=1 \\
x^{+}=A_{J} x \\
\tau^{+}=0
\end{array}\right\} \text { if } x \in \mathcal{J} \text { and } \tau \geq \rho
$$

with

$$
\begin{gathered}
A_{F}=\left[\begin{array}{cc|c}
A_{p}+B_{p} D_{c} C_{p} & B_{p} C_{c} & 0 \\
B_{c} C_{p} & A_{c} & C_{a} \\
\hline 0 & 0 & A_{a}
\end{array}\right]=\left[\begin{array}{c|c}
A & R C_{a} \\
\hline 0 & A_{a}
\end{array}\right], B_{F}=\left[\begin{array}{c}
B_{p} \\
D_{a} \\
\hline B_{a}
\end{array}\right]=\left[\begin{array}{c}
B+R D_{a} \\
B_{a}
\end{array}\right] \\
B_{2}=\left[\begin{array}{c}
B_{w} \\
0 \\
\hline 0
\end{array}\right]=\left[\begin{array}{c}
\mathbb{B}_{w} \\
\hline 0
\end{array}\right], B=\left[\begin{array}{c}
B_{p} \\
0
\end{array}\right], R=\left[\begin{array}{c}
0 \\
I_{n_{c}}
\end{array}\right], C=\left[\begin{array}{cc}
C_{p} & 0 \mid 0
\end{array}\right]=\left[\begin{array}{cc}
C_{1} \mid 0
\end{array}\right] \\
C_{2}=\left[\begin{array}{ll}
C_{z}+D_{z} D_{c} C_{p} & D_{z} C_{c} \mid 0
\end{array}\right]=\left[\mathbb{C}_{z} \mid 0\right]
\end{gathered}
$$

Matrix $A_{J}$, the flow and jump sets $\mathcal{F}$ and $\mathcal{J}$ will be precisely defined from several ways in the sequel. System (9) clearly lies in the set of hybrid and nonlinear control systems. To ensure that the solutions are in the flow set after a jump (see [12]), we adopt the following assumption (see [21]).

Assumption 1 For system (9), the reset matrix $A_{J}$ is such that $x \in \mathcal{J} \Longrightarrow x^{+} \in \mathcal{F}$.

\subsection{Flow and jump sets case 1}

We consider the case where the flow and jump sets are defined from the classical reset rule depending on the sign of the product between $y$ (output of the plant) and $y_{c}$ (output of the controller) (see for example [32], [20]). Hence, the sets $\mathcal{F}$ and $\mathcal{J}$ are defined by:

$$
\begin{aligned}
\mathcal{F} & =\left\{x \in \mathbb{R}^{n} ; x^{\prime} M x \geq 0\right\} \\
\mathcal{J} & =\left\{x \in \mathbb{R}^{n} ; x^{\prime} M x \leq 0\right\}
\end{aligned}
$$

In (11), matrix $M$ is a reset matrix of appropriate dimensions satisfying $M=Q^{\prime} \mathcal{M} Q$ with:

$$
\begin{aligned}
\mathcal{M} & =\left[\begin{array}{cc}
0 & -1 \\
-1 & 0
\end{array}\right] \in \mathbb{R}^{2 \times 2} \\
Q & =\left[\begin{array}{ccc}
C_{p} & 0 & 0 \\
D_{c} C_{p} & C_{c} & 0
\end{array}\right]=\left[\begin{array}{l}
C \\
K
\end{array}\right]=\left[\begin{array}{ll}
C_{1} & 0 \\
K_{1} & 0
\end{array}\right]=\left[\begin{array}{ll}
Q_{1} & 0
\end{array}\right] \in \mathbb{R}^{2 \times n}
\end{aligned}
$$


In the current case, two possible structures for the desired matrix $A_{J} \in \mathbb{R}^{n \times n}$ satisfying Assumption 1 can be considered :

$$
A_{J}=\left[\begin{array}{ccc}
I_{n_{p}} & 0 & 0 \\
0 & 0 & 0 \\
0 & 0 & 0
\end{array}\right]=\left[\begin{array}{cc}
A_{J 0} & 0 \\
0 & 0
\end{array}\right] \text { or } A_{J}=\left[\begin{array}{ccc}
I_{n_{p}} & 0 & 0 \\
0 & 0 & 0 \\
0 & 0 & I_{n_{a}}
\end{array}\right]=\left[\begin{array}{cc}
A_{J 0} & 0 \\
0 & I_{n_{a}}
\end{array}\right]
$$

That means that both the state of the controller and of the anti-windup compensator are reset (first structure) or only the state of the controller is reset (second structure).

Let us first suppose that the anti-windup compensator (5) is given (that is matrices $A_{a}, B_{a}, C_{a}, D_{a}$ are known). The following general result can be stated in the regional (local) context.

Proposition 2 If there exist two symmetric positive definite matrices $W \in \mathbb{R}^{n \times n}, M_{1} \in \mathbb{R}^{2 \times 2}$, a matrix $Z \in \mathbb{R}^{1 \times n}$ and positive scalars $S, \delta, \gamma, \tau_{F}, \tau_{R}$ satisfying

$$
\begin{aligned}
& {\left[\begin{array}{ccccc}
W A_{F}^{\prime}+A_{F} W & B_{F} S-Z^{\prime} & B_{2} & W C_{2}^{\prime} & \tau_{F} W Q^{\prime} \\
\star & -2 S & 0 & S D_{z}^{\prime} & 0 \\
\star & \star & -I & 0 & 0 \\
\star & \star & \star & -\gamma I & 0 \\
\star & \star & \star & \star & -\tau_{F} M_{1}^{-1}
\end{array}\right]<0} \\
& {\left[\begin{array}{cc}
W & W K^{\prime}-Z^{\prime} \\
\star & \delta u_{0}^{2}
\end{array}\right] \geq 0} \\
& {\left[\begin{array}{ccc}
-W & W A_{J}^{\prime} & \tau_{R} W Q^{\prime} \\
\star & -W & 0 \\
\star & \star & -\tau_{R} M_{1}^{-1}
\end{array}\right] \leq 0} \\
& -M_{1} \leq \mathcal{M} \leq M_{1}
\end{aligned}
$$

then:

1. When $w=0$, the nonlinear closed-loop system (9), with $A_{J}$ as in (13), remains stable for any initial condition belonging to $\mathcal{E}(P, \delta)$, with

$$
\mathcal{E}(P, \delta)=\left\{x \in \mathbb{R}^{n} ; x^{\prime} P x \leq \delta^{-1}\right\}, P=W^{-1}
$$

2. When $w \neq 0$, for $x(0)=0$,

- the closed-loop trajectories remain bounded in $\mathcal{E}(P, \delta)$,

- the map from $z$ to $w$ is finite gain $\mathcal{L}_{2}$ stable with

$$
\int_{0}^{T} z(t)^{\prime} z(t) d t \leq \gamma \int_{0}^{T} w(t)^{\prime} w(t) d t, \forall T \geq 0
$$

Proof. Consider the quadratic Lyapunov function $V(x)=x^{\prime} P x$, with $P=P^{\prime}=W^{-1}>0$ and the following change of variables: $G W=Z$. The satisfaction of relation (15) guarantees that the ellipsoid $\mathcal{E}(P, \delta)$ is included in the polyhedral set $S\left(u_{0}\right)=\left\{x \in \mathbb{R}^{n} ;-u_{0} \leq(K-G) x \leq u_{0}\right\}$. By using Lemma 1 in [28], we can verify that $-\phi(K x) S^{-1}(\phi(K x)+G x) \geq 0$, with $S$ a positive scalar, provided that $x \in S\left(u_{0}\right)$. Thus, for any $x \in \mathcal{E}(P, \delta)$ one gets: $\dot{V}(x)+\frac{1}{\gamma} z^{\prime} z-w^{\prime} w \leq \dot{V}(x)+\frac{1}{\gamma} z^{\prime} z-w^{\prime} w-2 \phi(K x) S^{-1}(\phi(K x)+G x)$. 
By developing the right-hand term of this inequality one obtains:

$$
\left[\begin{array}{c}
W^{-1} x \\
S^{-1} \phi(K x) \\
w
\end{array}\right]^{\prime}\left\{\left[\begin{array}{ccc}
W A_{F}^{\prime}+A_{F} W & B_{F} S-Z^{\prime} & B_{2} \\
\star & -2 S & 0 \\
\star & \star & -I
\end{array}\right]+\frac{1}{\gamma}\left[\begin{array}{c}
W C_{2}^{\prime} \\
S D_{z}^{\prime} \\
0
\end{array}\right]\left[\begin{array}{ccc}
C_{2} W & D_{z} S & 0
\end{array}\right]\right\}\left[\begin{array}{c}
W^{-1} x \\
S^{-1} \phi(K x) \\
w
\end{array}\right]
$$

By using the Schur complement it follows:

$$
\mathcal{L}=\left[\begin{array}{cccc}
W A_{F}^{\prime}+A_{F} W & B_{F} S-Z^{\prime} & B_{2} & W C_{2}^{\prime} \\
\star & -2 S & 0 & S D_{z}^{\prime} \\
\star & \star & -I & 0 \\
\star & \star & \star & -\gamma I
\end{array}\right]
$$

Let us check that $\mathcal{L}<0$ when the system (9) is flowing. Let $\tilde{t}$ be a time of flow. Two cases may occur: $x(\tilde{t}) \in \mathcal{F}$ or $\tau \leq \rho$.

Case 1. If $\bar{x}(\tilde{t}) \in \mathcal{F}$ then $\dot{V}(x)+\frac{1}{\gamma} z^{\prime} z-w^{\prime} w<0$ as soon as $\mathcal{L}<0$ for all $x \in \mathcal{F}$. Hence by using the S-procedure, this is equivalent to satisfy:

$$
\mathcal{L}_{0}=\left[\begin{array}{cccc}
W A_{F}^{\prime}+A_{F} W+\tau_{F} W Q^{\prime} \mathcal{M} Q W & B_{F} S-Z^{\prime} & B_{2} & W C_{2}^{\prime} \\
\star & -2 S & 0 & S D_{z}^{\prime} \\
\star & \star & -I & 0 \\
\star & \star & \star & -\gamma I
\end{array}\right]<0
$$

Hence from relation (17), matrix $\mathcal{L}_{0}$ can be over-bounded as follows:

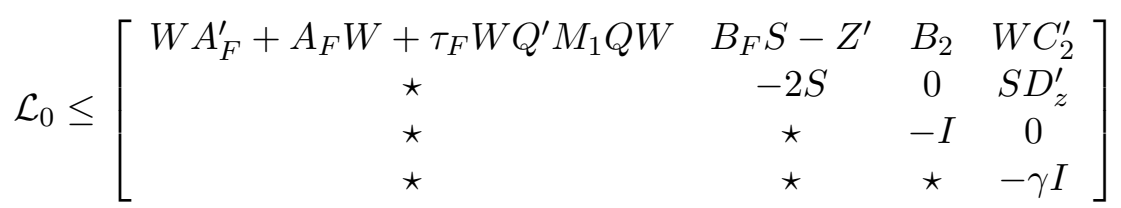

By using the Schur complement, the right-hand term of this inequality reads:

$$
\mathcal{L}_{1}=\left[\begin{array}{ccccc}
W A_{F}^{\prime}+A_{F} W & B_{F} S-Z^{\prime} & B_{2} & W C_{2}^{\prime} & \tau_{F} W Q^{\prime} \\
\star & -2 S & 0 & S D_{z}^{\prime} & 0 \\
\star & \star & -I & 0 & 0 \\
\star & \star & \star & -\gamma I & 0 \\
\star & \star & \star & \star & -\tau_{F} M_{1}^{-1}
\end{array}\right]
$$

If one ensures $\mathcal{L}_{1}<0$, which holds if (14) is verified, then it follows that $\dot{V}(x)+\frac{1}{\gamma} z^{\prime} z-w^{\prime} w<0$.

Case 2. Suppose that one gets $\tau \leq \rho$. Consider $\underline{t} \leq \tilde{t}$ the smallest time where we are flowing in $[\underline{t}, \tilde{t}]$. We have $x(\underline{t}) \in \mathcal{F}$ since either $\underline{t}=0$, or we have a jump at $\underline{t}$ (and due to Assumption 1 for all $x \in \mathcal{J}$, we have $\left.A_{J} x \in \mathcal{F}\right)$. Then, there exists a positive scalar $\eta$ such that, for all $t \in[\underline{t}, \rho)$, we have $x(t) \in \mathcal{F}_{\eta}$ with $\mathcal{F}_{\eta}=\left\{x \in \mathbb{R}^{n} ; x^{\prime} M x+\eta x^{\prime} x \geq 0\right\}$. Thus, we remain in $\mathcal{E}(P, \delta)$ and we have $\dot{V}(x(t))+\frac{1}{\gamma} z^{\prime} z-w^{\prime} w<0$ for all $t \in\left[\underline{t}, \rho\right.$ ) as soon as $\mathcal{L}<0$ (with $\mathcal{L}$ defined in (20)) for all $x \in \mathcal{F}_{\eta}$, which is equivalent to verify:

$$
\left[\begin{array}{cccc}
W A_{F}^{\prime}+A_{F} W+\tau_{F} W\left(Q^{\prime} \mathcal{M} Q+\eta I_{n}\right) W & B_{F} S-Z^{\prime} & B_{2} & W C_{2}^{\prime} \\
\star & -2 S & 0 & S D_{z}^{\prime} \\
\star & \star & -I & 0 \\
\star & \star & \star & -\gamma I
\end{array}\right]<0
$$

Moreover, note that $\eta \rightarrow 0$ as $\rho \rightarrow 0$. Therefore, by considering a small enough value for $\rho$, we can consider small enough $\eta$ to be neglected and it follows that the satisfaction of relation (14) guarantees that inequality (21) holds. 
Moreover, we want also to prove that $V\left(x^{+}\right)-V(x) \leq 0$ for $x \in \mathcal{J}$. This condition holds if the following relation is satisfied:

$$
x^{\prime}\left(A_{J}^{\prime} P A_{J}-P\right) x-\tau_{R} x^{\prime} M x=x^{\prime}\left(A_{J}^{\prime} P A_{J}-P\right) x-\tau_{R} x^{\prime} Q^{\prime} \mathcal{M} Q x \leq 0
$$

which reads, by using the Schur complement upon pre- and post-multiplying (22) by $W$ :

$$
\left[\begin{array}{cc}
-W-\tau_{R} W Q^{\prime} \mathcal{M} Q W & W A_{J}^{\prime} \\
\star & -W
\end{array}\right] \leq 0
$$

From relation (17) one can over-bound the term $-\tau_{R} W Q^{\prime} \mathcal{M} Q W$ by $\tau_{R} W Q^{\prime} M_{1} Q W$. Thus, by using the Schur complement, condition (23) can be rewritten as:

$$
\left[\begin{array}{ccc}
-W & W A_{J}^{\prime} & \tau_{R} W Q^{\prime} \\
\star & -W & 0 \\
\star & \star & -\tau_{R} M_{1}^{-1}
\end{array}\right] \leq 0
$$

Hence, the satisfaction of relations (14), (15), (16) and (17) ensures that along the trajectories of the hybrid system (9) the Lyapunov function satisfies

$$
\begin{array}{lll}
\dot{V}(x(t))+\frac{1}{\gamma} z^{\prime} z-w^{\prime} w<0 & \text { if } & x \in \mathcal{F} \\
\Delta V(x) \leq 0 & \text { if } & x \in \mathcal{J}
\end{array}
$$

for all $w$ satisfying (2). Hence, it follows that

- for $x(0)=0$, one gets $V(0)=0$ and $V(T)=x(T)^{\prime} P x(T) \leq \int_{0}^{T} w(t)^{\prime} w(t) d t \leq \delta^{-1}, \forall T \geq 0$. In other words, the trajectories of the closed-loop system (9) do not leave the set $\mathcal{E}(P, \delta)$.

- When $T \rightarrow \infty$, one gets $\int_{0}^{\infty} z(t)^{\prime} z(t) d t \leq \gamma \int_{0}^{\infty} w(t)^{\prime} w(t) d t$, therefore the $\mathcal{L}_{2}$-gain of the system is less than $\gamma$.

- If $w=0$ then one obtains $\dot{V}(x)<-\frac{1}{\gamma} x^{\prime} P x<0$. The nonlinear closed-loop system (9) with $A_{J}$ defined from (13) remains stable for any initial condition belonging to $\mathcal{E}(P, \delta)$.

This completes the proof of Proposition 2.

Let us focus on the synthesis issue as stated in Problem 1. It is clear that it is not possible to determine simultaneously the analysis variable $W$ and the anti-windup matrices $A_{a}, B_{a}, C_{a}$ and $D_{a}$ from the conditions of Proposition 2. By considering the case where $n_{a}=n_{p}+n_{c}$, these conditions can be simplified. In particular, different frameworks can be adapted in order to obtain quasi-convex or convex conditions in the case $n_{a}=n+n_{c}$ : one can, for example, cite the framework used in [3], [9], based on the projection lemma, or that one based on some change of variables [26], [2]. For the ease of the presentation, we choose the second framework above mentioned.

The following theorem exhibits solution in the sense to design the anti-windup compensator satisfying Proposition 2.

Theorem 3 If there exist three symmetric positive definite matrices $X \in \mathbb{R}^{\left(n_{p}+n_{c}\right) \times\left(n_{p}+n_{c}\right)}, Y \in \mathbb{R}^{\left(n_{p}+n_{c}\right) \times\left(n_{p}+n_{c}\right)}$, $M_{1} \in \mathbb{R}^{2 \times 2}$, matrices $Z_{1} \in \mathbb{R}^{1 \times\left(n_{p}+n_{c}\right)}, \mathcal{Z} \in \mathbb{R}^{1 \times\left(n_{p}+n_{c}\right)}, \Omega_{1} \in \mathbb{R}^{\left(n_{p}+n_{c}\right) \times\left(n_{p}+n_{c}\right)}, \Omega_{2} \in \mathbb{R}^{\left(n_{p}+n_{c}\right) \times 1}$, $\Omega_{3} \in \mathbb{R}^{n_{c} \times\left(n_{p}+n_{c}\right)}, \Omega_{4} \in \mathbb{R}^{n_{c} \times 1}$, positive scalars $S, \tau_{F}, \tau_{R}, \delta$, $\gamma$ such that relation (17) and the following conditions hold:

$$
\left[\begin{array}{cccccc}
X A^{\prime}+A X & X A^{\prime}+A Y+R \Omega_{3}+\Omega_{1} & B S+R \Omega_{4}+\Omega_{2}-\mathcal{Z}^{\prime} & \mathbb{B}_{w} & X \mathbb{C}_{z}^{\prime} & \tau_{F} X Q_{1}^{\prime} \\
\star & A Y+Y A^{\prime}+R \Omega_{3}+\Omega_{3}^{\prime} R^{\prime} & B S+R \Omega_{4}-Z_{1}^{\prime} & \mathbb{B}_{w} & Y \mathbb{C}_{z}^{\prime} & \tau_{F} Y Q_{1}^{\prime} \\
\star & \star & -2 S & 0 & S D_{z}^{\prime} & 0 \\
\star & \star & \star & -I & 0 & 0 \\
\star & \star & \star & \star & -\gamma I & 0 \\
\star & \star & \star & \star & \star & -\tau_{F} M_{1}^{-1}
\end{array}\right]<0
$$




$$
\begin{gathered}
{\left[\begin{array}{cccc}
X & X & X K_{1}^{\prime}-\mathcal{Z}^{\prime} \\
\star & Y & Y K_{1}^{\prime}-Z_{1}^{\prime} \\
\star & \star & \delta u_{0}^{2}
\end{array}\right] \geq 0} \\
{\left[\begin{array}{ccccc}
-X & -X & X A_{J 0}^{\prime} & X A_{J 0}^{\prime} & \tau_{R} X Q_{1}^{\prime} \\
\star & -Y & Y A_{J 0}^{\prime} & Y A_{J 0}^{\prime} & \tau_{R} Y Q_{1}^{\prime} \\
\star & \star & -X & -X & 0 \\
\star & \star & \star & -Y & 0 \\
\star & \star & \star & \star & -\tau_{R} M_{1}^{-1}
\end{array}\right] \leq 0}
\end{gathered}
$$

then the anti-windup controller defined by

$$
\begin{aligned}
A_{a} & =\left(\mathcal{U}^{\prime}\right)^{-1} X^{-1} \Omega_{1} N^{-1} \\
B_{a} & =\left(\mathcal{U}^{\prime}\right)^{-1} X^{-1} \Omega_{2} S^{-1} \\
C_{a} & =\Omega_{3} N^{-1} \\
D_{a} & =\Omega_{4} S^{-1}
\end{aligned}
$$

where matrices $\mathcal{U}, N$ verify $N^{\prime} \mathcal{U}=I-Y X^{-1}$, is solution to Problem 1, that is,

1. When $w=0$, the nonlinear closed-loop system (9), with $A_{J}$ defined as the first structure in (13), remains stable for any initial condition belonging to the set $\mathcal{E}(P, \delta)$, defined as in (18) with

$$
P=W^{-1}=\left[\begin{array}{cc}
X^{-1} & \mathcal{U}^{\prime} \\
\star & F
\end{array}\right] ; W=\left[\begin{array}{cc}
Y & N^{\prime} \\
\star & H
\end{array}\right]
$$

2. When $w \neq 0$, for $x(0)=0$,

- the closed-loop trajectories remain bounded in $\mathcal{E}(P, \delta)$,

- the map from $z$ to $w$ is finite gain $\mathcal{L}_{2}$ stable and satisfies (19).

Proof. Let us define: $W=\left[\begin{array}{cc}Y & N^{\prime} \\ \star & H\end{array}\right]$ and $P=W^{-1}=\left[\begin{array}{cc}X^{-1} & \mathcal{U}^{\prime} \\ \star & F\end{array}\right]$, where $X, Y, N, \mathcal{U}, F$ and $H$ are decision variables of appropriate dimensions. Then, it follows: $Y X^{-1}+N^{\prime} \mathcal{U}=I, Y \mathcal{U}^{\prime}+N^{\prime} F=0$, $N X^{-1}+H \mathcal{U}=0$ and $N \mathcal{U}^{\prime}+H F=I$. Similarly to [26], we use the matrix $\Gamma=\left[\begin{array}{cc}I & I \\ \mathcal{U} X & 0\end{array}\right]$. From this, one gets:

$$
\begin{aligned}
& \Gamma^{\prime} W=\left[\begin{array}{cc}
X & 0 \\
Y & N^{\prime}
\end{array}\right] ; \Gamma^{\prime} W \Gamma=\left[\begin{array}{cc}
X & X \\
X & Y
\end{array}\right] \\
& \Gamma^{\prime} W A_{F}^{\prime} \Gamma=\left[\begin{array}{cc}
X A^{\prime} & X A^{\prime} \\
Y A^{\prime}+N^{\prime} C_{a}^{\prime} R^{\prime}+N^{\prime} A_{a}^{\prime} \mathcal{U} X & Y A^{\prime}+N^{\prime} C_{a}^{\prime} R^{\prime}
\end{array}\right] \\
& \Gamma^{\prime}\left(B_{F} S-Z^{\prime}\right)=\left[\begin{array}{c}
B S+R D_{a} S+X \mathcal{U}^{\prime} B_{a} S-\left(Z_{1}^{\prime}+X \mathcal{U}^{\prime} Z_{2}^{\prime}\right) \\
B S+R D_{a} S-Z_{1}^{\prime}
\end{array}\right] \\
& \Gamma^{\prime} B_{2}=\left[\begin{array}{c}
\mathbb{B}_{w} \\
\mathbb{B}_{w}
\end{array}\right] ; \Gamma^{\prime} W C_{2}^{\prime}=\left[\begin{array}{c}
X \mathbb{C}_{z}^{\prime} \\
Y \mathbb{C}_{z}^{\prime}
\end{array}\right] ; \Gamma^{\prime} W Q^{\prime}=\left[\begin{array}{c}
X Q_{1}^{\prime} \\
Y Q_{1}^{\prime}
\end{array}\right]
\end{aligned}
$$

where the variable $Z$ can be partitioned as $Z=\left[\begin{array}{ll}Z_{1} & Z_{2}\end{array}\right] \in \mathbb{R}^{1 \times n}$, with $Z_{1} \in \mathbb{R}^{1 \times\left(n_{p}+n_{c}\right)}$ and $Z_{2} \in$ $\mathbb{R}^{1 \times\left(n_{p}+n_{c}\right)}\left(\right.$ since $\left.n_{a}=n_{p}+n_{c}\right)$.

Hence, relation (25) is obtained by pre- and post-multiplying relation (14) by $\operatorname{diag}\left(\Gamma^{\prime}, I, I, I, I\right)$ and by $\operatorname{diag}(\Gamma, I, I, I, I)$, respectively and by using the following change of variables:

$$
\Omega_{1}=X \mathcal{U}^{\prime} A_{a} N ; \Omega_{2}=X \mathcal{U}^{\prime} B_{a} S ; \Omega_{3}=C_{a} N ; \Omega_{4}=D_{a} S ; \mathcal{Z}=Z_{1}+Z_{2} \mathcal{U} X
$$


By the same way, relation (26) is obtained by pre- and post-multiplying relation (15) by $\operatorname{diag}\left(\Gamma^{\prime}, I\right)$ and by $\operatorname{diag}(\Gamma, I)$ and by using the change of variables $(30)$.

Finally, relation (27) is obtained by pre- and post-multiplying relation (16) by $\operatorname{diag}\left(\Gamma^{\prime}, I, I\right)$ and by $\operatorname{diag}(\Gamma, I, I)$ and by considering the first structure of matrix $A_{J}$ given in (13). Then, it follows:

$$
\Gamma^{\prime} W A_{J}^{\prime} \Gamma=\left[\begin{array}{cc}
X A_{J 0}^{\prime} & X A_{J 0}^{\prime} \\
Y A_{J 0}^{\prime} & Y A_{J 0}^{\prime}
\end{array}\right]
$$

Similar arguments regarding flow and jump sets are used as in the proof of Proposition 2.

Remark 4 If the second structure for matrix $A_{J}$ given in (13) is used, relation (27) in Theorem 3 is slightly modified: the block (2,3) is changed as $Y A_{J 0}^{\prime}+X-Y$.

Remark 5 When the plant is asymptotically stable (i.e. matrix $A_{p}$ is Hurwitz), it is possible to seek the assurance of the global stability of the closed-loop system. The closed-loop system is finite gain $\mathcal{L}_{2}$ stable for any admissible $w$ satisfying (2) and any initial condition $x_{0} \in \mathbb{R}^{n}$, i.e. the origin of the system is globally asymptotically stable and one gets:

$$
\int_{0}^{T} z(t)^{\prime} z(t) d t \leq \gamma \int_{0}^{T} w(t)^{\prime} w(t) d t+\gamma x_{0}^{\prime} P x_{0}, \forall T \geq 0, \forall x_{0} \in \mathbb{R}^{n}
$$

Hence, the global asymptotic stability can be addressed by considering $Z=K W$ in Proposition 2. In this case, one chooses $\mathcal{Z}=Z_{1}=K_{1} W$ and therefore one replaces condition (26) by $Y-X>0$ in Theorem 3.

\subsection{Flow and jump sets case 2}

We consider the case where the flow and jump sets are defined from the region of stability $\mathcal{E}(P, \delta)=\{x \in$ $\left.\mathbb{R}^{n} ; x^{\prime} P x \leq \delta^{-1}\right\}$ with $P=P^{\prime}>0, P \in \mathbb{R}^{n \times n}$. By using similar tools as in Theorem 3 , the following theorem provides convex conditions to exhibit both the suitable anti-windup matrices $A_{a}, B_{a}, C_{a}, D_{a}$ and the reset matrix $A_{J}$.

Let us first define the structure of the desired matrix $A_{J}$ :

$$
A_{J}=\left[\begin{array}{cc}
A_{J 0} & 0 \\
0 & 0
\end{array}\right]+E_{r} \Theta F_{r}
$$

with

$$
\begin{aligned}
& A_{J 0}=\left[\begin{array}{cc}
I_{n_{p}} & 0 \\
0 & 0
\end{array}\right] ; E_{r}=\left[\begin{array}{cc}
0 & 0 \\
I & 0 \\
0 & I
\end{array}\right] ; F_{r}=\left[\begin{array}{ccc}
C_{p} & 0 & 0 \\
0 & I & 0 \\
0 & 0 & I
\end{array}\right]=\left[\begin{array}{cc}
C_{1} & 0 \\
R^{\prime} & 0 \\
0 & I
\end{array}\right] \\
& \Theta=\left[\begin{array}{ccc}
A_{r 1} & B_{r 1} & C_{r 1} \\
A_{r 2} & B_{r 2} & C_{r 2}
\end{array}\right]=\left[\begin{array}{lll}
A_{r} & B_{r} & C_{r}
\end{array}\right]
\end{aligned}
$$

In the proposed structure (33), matrices $A_{r} \in \mathbb{R}^{\left(n_{p}+2 n_{c}\right) \times 1}, B_{r} \in \mathbb{R}^{\left(n_{p}+2 n_{c}\right) \times n_{c}}, C_{r} \in \mathbb{R}^{\left(n_{p}+2 n_{c}\right) \times\left(n_{p}+n_{c}\right)}$ are decision variables, which correspond to the jump equations:

$$
\begin{aligned}
& x_{p}^{+}=x_{p} \\
& x_{c}^{+}=A_{r 1} C_{p} x_{p}+B_{r 1} x_{c}+C_{r 1} x_{a} \\
& x_{a}^{+}=A_{r 2} C_{p} x_{p}+B_{r 1} x_{c}+C_{r 1} x_{a}
\end{aligned}
$$

Furthermore, the flow and jump sets are defined as

$$
\begin{aligned}
\mathcal{F} & =\mathcal{E}(P, \delta) \\
\mathcal{J} & =\operatorname{clos}\left(\mathbb{R}^{n} \backslash \mathcal{E}(P, \delta)\right)
\end{aligned}
$$

where $\operatorname{clos}($.$) stands for the closure.$ 
Theorem 6 If there exist four symmetric positive definite matrices $X \in \mathbb{R}^{\left(n_{p}+n_{c}\right) \times\left(n_{p}+n_{c}\right)}, Y \in \mathbb{R}^{\left(n_{p}+n_{c}\right) \times\left(n_{p}+n_{c}\right)}$, $P_{11} \in \mathbb{R}^{\left(n_{p}+n_{c}\right) \times\left(n_{p}+n_{c}\right)}, P_{13} \in \mathbb{R}^{\left(n_{p}+n_{c}\right) \times\left(n_{p}+n_{c}\right)}$, matrices $Z_{1} \in \mathbb{R}^{1 \times\left(n_{p}+n_{c}\right)}, \mathcal{Z} \in \mathbb{R}^{1 \times\left(n_{p}+n_{c}\right)}, P_{12} \in$ $\mathbb{R}^{\left(n_{p}+n_{c}\right) \times\left(n_{p}+n_{c}\right)}, \Omega_{1} \in \mathbb{R}^{\left(n_{p}+n_{c}\right) \times\left(n_{p}+n_{c}\right)}, \Omega_{2} \in \mathbb{R}^{\left(n_{p}+n_{c}\right) \times 1}, \Omega_{3} \in \mathbb{R}^{n_{c} \times\left(n_{p}+n_{c}\right)}, \Omega_{4} \in \mathbb{R}^{n_{c} \times 1}, \Omega_{5} \in$ $\mathbb{R}^{\left(2 n_{c}+n_{p}\right) \times 1}, \Omega_{6} \in \mathbb{R}^{\left(2 n_{c}+n_{p}\right) \times n_{c}}, \Omega_{7} \in \mathbb{R}^{\left(2 n_{c}+n_{p}\right) \times\left(n_{c}+n_{p}\right)}$, positive scalars $S, \delta, \gamma$ satisfying

$$
\begin{aligned}
& {\left[\begin{array}{ccccc}
X A^{\prime}+A X & X A^{\prime}+A Y+R \Omega_{3}+\Omega_{1} & B S+R \Omega_{4}+\Omega_{2}-\mathcal{Z}^{\prime} & \mathbb{B}_{w} & X \mathbb{C}_{z}^{\prime} \\
\star & A Y+Y A^{\prime}+R \Omega_{3}+\Omega_{3}^{\prime} R^{\prime} & B S+R \Omega_{4}-Z_{1}^{\prime} & \mathbb{B}_{w} & Y \mathbb{C}_{z}^{\prime} \\
\star & \star & -2 S & 0 & S D_{z}^{\prime} \\
\star & \star & \star & -I & 0 \\
\star & \star & \star & \star & -\gamma I
\end{array}\right]<0} \\
& {\left[\begin{array}{ccc}
X & X & X K_{1}^{\prime}-\mathcal{Z}^{\prime} \\
\star & Y & Y K_{1}^{\prime}-Z_{1}^{\prime} \\
\star & \star & \delta u_{0}^{2}
\end{array}\right] \geq 0} \\
& {\left[\begin{array}{cccc}
P_{11} & P_{12} & A_{J 0}^{\prime}+C_{1}^{\prime} \Omega_{5}^{\prime}+R \Omega_{6}^{\prime} & A_{J 0}^{\prime} \\
\star & P_{13} & \Omega_{7}^{\prime} & 0 \\
\star & \star & X & X \\
\star & \star & \star & Y
\end{array}\right] \geq 0}
\end{aligned}
$$

then the anti-windup controller defined by

$$
\begin{aligned}
A_{a} & =\left(\mathcal{U}^{\prime}\right)^{-1} X^{-1} \Omega_{1} N^{-1} \\
B_{a} & =\left(\mathcal{U}^{\prime}\right)^{-1} X^{-1} \Omega_{2} S^{-1} \\
C_{a} & =\Omega_{3} N^{-1} \\
D_{a} & =\Omega_{4} S^{-1}
\end{aligned}
$$

is solution to Problem 1 with $A_{J}$ defined from (32) with

$$
\begin{aligned}
& A_{r}=\left(\mathcal{U}^{\prime}\right)^{-1} X^{-1} \Omega_{5} \\
& B_{r}=\left(\mathcal{U}^{\prime}\right)^{-1} X^{-1} \Omega_{6} \\
& C_{r}=\left(\mathcal{U}^{\prime}\right)^{-1} X^{-1} \Omega_{7}
\end{aligned}
$$

where matrices $\mathcal{U}, N$ verify $N^{\prime} \mathcal{U}=I-Y X^{-1}$. In other words:

1. When $w=0$, the nonlinear closed-loop system (9) remains stable for any initial condition belonging to the set $\mathcal{E}(P, \delta) \cup \mathcal{E}\left(P_{1}, \delta\right)$, where $\mathcal{E}(P, \delta)$ is defined as in (18) and $\mathcal{E}\left(P_{1}, 1\right)=\left\{x \in \mathbb{R}^{n} ; x^{\prime} P_{1} x \leq \delta^{-1}\right\}$ with

$$
P=W^{-1}=\left[\begin{array}{cc}
X^{-1} & \mathcal{U}^{\prime} \\
\star & F
\end{array}\right] ; W=\left[\begin{array}{cc}
Y & N^{\prime} \\
\star & H
\end{array}\right] ; P_{1}=\left[\begin{array}{cc}
P_{11} & P_{12} \\
\star & P_{13}
\end{array}\right]
$$

2. When $w \neq 0$, for $x(0)=0$,

- the closed-loop trajectories remain bounded in $\mathcal{E}(P, \delta)$,

- the map from $z$ to $w$ is finite gain $\mathcal{L}_{2}$ stable and satisfies (19).

Proof. This proof mimicks in part that ones of Proposition 2 and Theorem 3. Similar definitions and changes of variables as in the proof of Theorem 3 are used to derive the conditions in the continuous part (flow case).

Let us focus on the discrete part (jump case) of the proof. We want to verify that $x_{0}^{+} \in \mathcal{E}(P, \delta)$ if $x_{0} \in \mathcal{E}\left(P_{1}, \delta\right)$, that is,

$$
x_{0}^{\prime} A_{J}^{\prime} P A_{J} x_{0} \leq \delta^{-1} \text { if } x_{0}^{\prime} P_{1} x_{0} \leq \delta^{-1}
$$


By using the S-procedure, it follows that this condition will be satisfied if one gets: $\delta\left(P_{1}-A_{J}^{\prime} P A_{J}\right) \geq 0$, or equivalently, $P_{1}-A_{J}^{\prime} P A_{J} \geq 0$. From the definition of $A_{J}$ given by (32)-(33) and by applying the Schur complement one gets the following relation:

$$
\left[\begin{array}{cc}
P_{1} & \left(A_{J}+E_{r} \Theta F_{r}\right)^{\prime} \\
\star & W
\end{array}\right] \geq 0
$$

Hence, relation (38) is obtained by pre- and post-multiplying relation $(42)$ by $\operatorname{diag}\left(I, \Gamma^{\prime}\right)$ and by $\operatorname{diag}(I, \Gamma)$, respectively and by using the following change of variables:

$$
\Omega_{5}=X \mathcal{U}^{\prime} A_{r} ; \Omega_{6}=X \mathcal{U}^{\prime} B_{r} ; \Omega_{7}=X \mathcal{U}^{\prime} C_{r}
$$

with $\Gamma=\left[\begin{array}{cc}I & I \\ \mathcal{U} X & 0\end{array}\right]$

Remark 7 The reset controller defined from (34) uses the measured output of the plant ( $\left.y=C_{p} x_{p}\right)$, and the states $x_{c}$ and $x_{a}$ of the controller and of the anti-windup compensator. Moreover, the flow and jump sets defining the reset rules in (35) depend on the complete state $x=\left[\begin{array}{lll}x_{p}^{\prime} & x_{c}^{\prime} & x_{a}^{\prime}\end{array}\right]^{\prime}$ along time. This requires that all system states are measurable. States $x_{c}$ and $x_{a}$ can be easily obtained by taking care during controller implementation. However, that is not the case for $x_{p}$ since by definition of system (1) only $C_{p} x_{p}$ is actually measured. Hence, a next step could be for example to use an observer and to redefine adequately the flow and jump sets from the observed state $\hat{x}_{p}$.

Remark 8 It is important to note that Theorem 6 could easily apply to MIMO systems contrary to Theorem 3. This is mainly due to the way chosen for describing the flow and jump sets.

\section{Computational and numerical issues}

Theorem 3 presents non-convex conditions: one of the nonlinearities (or non-convexity) is due to the presence of both $M_{1}$ and $M_{1}^{-1}$ in the conditions, $M_{1} \in \mathbb{R}^{2 \times 2}$ being a decision variable. Nevertheless, a way to overcome such a problem consists in fixing $M_{1}$ as a diagonal positive definite matrix. Then, a search for the optimal solution over a bi-dimensional grid (composed by the 2 elements of $M_{1}$ ) can be considered. A sub-case can be to choose $M_{1}=m_{1} I$ implying to use an iterative line search. An alternative solution consists in using a relaxation scheme, which translates the problem into a sequence of iterative LMI problems fixing $M_{1}$ or the other variables at each step. In this case, the convergence of the procedure is always ensured, but not necessarily to the global optimal value. Furthermore, the convergence value will depend on the initialization of $M_{1}$ in the iterative procedure. Then, a more adulterated approach based on a linearization algorithm, as detailed in [7], could allow to tackle the presence of both $M_{1}$ and $M_{1}^{-1}$ in the conditions.

The two other nonlinearities are due to the products $\tau_{F} X, \tau_{F} Y, \tau_{R} X, \tau_{R} Y$. Nevertheless, positive parameters $\tau_{F}$ and $\tau_{R}$ related to the use of the S-procedure may be a priori fixed. Although their numerical value may have an impact on the obtained solution, it remains limited and the a priori choice does not prevent from obtaining an admissible solution.

At this stage, it is interesting to point out that Theorem 6 provides LMI conditions.

Based on Theorems 3 and 6, several LMI-based optimization problems can be proposed to compute the anti-windup controller optimizing one of the following criteria: maximization of the $\mathcal{L}_{2}$ bound on the admissible disturbances (disturbance tolerance maximization which corresponds to minimize $\delta$ ); the minimization of the induced $\mathcal{L}_{2}$-gain between the disturbance $w$ and the regulated output $z$ (disturbance rejection maximization which corresponds to minimize $\gamma$ for a given $\delta$ ); or the maximization of the region where the asymptotic stability of the closed-loop system is ensured (maximization of the region of attraction). 
Remark 9 Conditions of Theorem 3 (and Theorem 6) imply the satisfaction $W A_{F}^{\prime}+A_{F} W<0$. Due to the structure of matrix $A_{F}$, this guarantees the asymptotic stability of the matrix $A_{a}$ of the anti-windup compensator. In the spirit of [25], it would then be possible to modify conditions of theorems in order to force the poles of the anti-windup controller (i.e., the poles of matrix $A_{a}$ ) to be located in a certain region.

\subsection{Maximization of the $\mathcal{L}_{2}$ bound}

In the case of Theorem 3, the following convex optimization problem can be considered: Fix $M_{1}$ for which (17) holds; Choose small enough $\tau_{F}>0$ and $\tau_{R}>0$; Solve $\min \delta$, subject to relations (25), (26), (27), with respect to the decision variables $X, Y, \delta, S, Z_{1}, \mathcal{Z}, \Omega_{1}, \Omega_{2}, \Omega_{3}, \Omega_{4}, \gamma$. In the case of Theorem 6 , we solve $\min \delta$, subject to relations (36), (37), (38), with respect to the decision variables $X, Y, P_{11}$, $P_{12}, P_{13}, \delta, S, Z_{1}, \mathcal{Z}, \Omega_{1}, \Omega_{2}, \Omega_{3}, \Omega_{4}, \Omega_{5}, \Omega_{6}, \Omega_{7}, \gamma$.

\subsection{Minimization of the induced $\mathcal{L}_{2}$-gain}

Given $\delta$, we want to solve the following optimization: $\min \gamma$ subject to relations of Theorem 3 or 6 .

\subsection{Maximization of the region of attraction}

At this aim, we consider the disturbance free case (i.e. $w=0$ ). Hence, a modified version of Theorem 3 or 6 is considered: in relation (25) or (36), the two lines and columns related to the disturbance and the output $z$ are removed. Different linear optimization criteria $J($.$) , associated to the size of \mathcal{E}(P, \delta)$, can be considered, like the volume, the size of the minor axis or the maximization of a scaling factor allowing to include a given shape in the ellipsoid [17], [13]. In particular it is interesting to address this problem in the plant space. For the sake of simplicity, in this case, we set $\delta=1$. Choose a set of interesting directions $v_{i} \in \mathbb{R}^{n_{p}}, i=1, \ldots, q$. Define $\tilde{v}_{i}=N_{a} v_{i} \in \mathbb{R}^{n_{p}+n_{c}}$, with $N_{a}=\left[\begin{array}{c}I_{n_{p}} \\ 0\end{array}\right]$, one considers the additional constraint $\mu-\left[\begin{array}{cc}\tilde{v}_{i}^{\prime} & 0\end{array}\right] P\left[\begin{array}{c}\tilde{v}_{i} \\ 0\end{array}\right] \geq 0, i=1, \ldots, q$, or equivalently from the definition of $P$ :

$$
\left[\begin{array}{cc}
\mu & v_{i}^{\prime} N_{a}^{\prime} \\
\star & X
\end{array}\right] \geq 0, i=1, \ldots, q
$$

Thus, we want to solve the following optimization problem: $\min \mu$ subject to (44) and modified relations of Theorems 3 or 6 .

\subsection{Reduced-order anti-windup compensator}

Let us now focus on a special case where the matrices $A_{a}$ and $C_{a}$ of the anti-windup compensator are a priori fixed. This allows not only to control the order of the anti-windup compensator but also to reduce the computational efforts. For example, in relation (14) of Proposition 2 the main nonlinearity involving $W, A_{a}$ and $C_{a}$ can be removed as soon as the matrices $A_{a}$ and $C_{a}$ of the anti-windup controller are fixed. The main difficulty however resides in choosing the matrices $A_{a}$ and $C_{a}$ adequately. According to the approach developed in [3] for instance (see also [31], [19]), this choice may be carried out by considering the poles of the anti-windup controller. These poles can be chosen by selecting a part of poles obtained in the full order design case $\left(n_{a}=n_{p}+n_{c}\right)$. Typically, the slow and fast dynamics are eliminated. Alternatively, an iterative procedure starting from the static case can be used. The list of poles is then progressively enriched until the gap between the full and reduced order cases becomes small enough. Note then that the order of the controller is now given by $n_{a}=n_{1}+2 n_{2}$, where $n_{1}$ and $n_{2}$ correspond 
respectively to real and complex poles and have to be chosen sufficiently small, so that $n_{a}<n_{p}+n_{c}$. The algorithm proposed in [19] is based on a different decomposition of the anti-windup controller using dyadic forms and the associated procedure requires the user to specify output pole directions which may not be trivial.

Another interesting way consists in imposing a particular architecture to the anti-windup compensator, quite different from the anti-windup scheme (5) and copying in part the plant as follows [30], [33], $[9]$ :

$$
\begin{aligned}
& \dot{x}_{a}=\left(A_{p}+B_{p} F_{a}\right) x_{a}+B_{p}\left(\operatorname{sat}\left(y_{c}+y_{a 1}\right)-\left(y_{c}+y_{a 1}\right)\right) \\
& y_{a 1}=F_{a} x_{a} \\
& y_{a 2}=C_{p} x_{a}
\end{aligned}
$$

leading to the modified controller:

$$
\begin{aligned}
& \dot{x}_{c}(t, j)=A_{c} x_{c}(t, j)+B_{c}\left(y(t)+y_{a 2}\right) \\
& y_{c}(t, j)=C_{c} x_{c}(t, j)+D_{c}\left(y(t)+y_{a 2}\right) \\
& \dot{\tau}(t, j)=1 \\
& \left.\begin{array}{l}
x_{c}\left(t_{j+1}, j+1\right)=0 \\
\tau\left(t_{j+1}, j+1\right)=0
\end{array}\right\} \text { if }\left(x_{p}, x_{c}, x_{a}\right) \in \mathcal{F} \text { or } \tau \leq \rho \\
&
\end{aligned}
$$

where $x_{a} \in \mathbb{R}^{n_{p}}, \operatorname{sat}\left(y_{c}+y_{a 1}\right)-\left(y_{c}+y_{a 1}\right), y_{a 2} \in \mathbb{R}^{1}, y_{a 1} \in \mathbb{R}^{1}$ are the state, the input and the outputs of the anti-windup compensator. Using the augmented state vector $x$, as defined in (7), a similar closed-loop system as described in (9) can be obtained. The anti-windup design then consists in synthesizing the gain $F_{a}$ such that matrix $A_{p}+B_{p} F_{a}$ is Hurwitz. From this, Proposition 2 can be used. Similarly to Section 3.3, we can adapt the conditions in the case where the flow and jump sets are defined from the region of stability.

Remark 10 The static anti-windup case can be considered: by choosing $n_{a}=0, A_{a}=0, B_{a}=0, C_{a}=0$ and by computing the gain $D_{a}$.

\subsection{Numerical examples}

We consider the system proposed in [14] characterized by the linear open-loop unstable system:

$$
\begin{aligned}
& \dot{x}_{p}=0.1 x_{p}+u \\
& y=z=x
\end{aligned}
$$

where $u$ satisfies relation (3) with $u_{0}=1$ and the stabilizing PI controller

$$
\begin{aligned}
& \dot{x}_{c}=-0.2 y \\
& y_{c}=x_{c}-2 y
\end{aligned}
$$

\subsubsection{Flow and jump set case 1}

We first aim at applying Theorem 3 to solve the optimization problem proposed in Section 4.3. System (47)-(48) is subject to a reset control law satisfying (11)-(12). We define the set of interesting directions, denoted $\Xi_{0}$, as a square region in $\mathbb{R}^{2}$ :

$$
\Xi_{0}=C o\left\{\left[\begin{array}{l}
1 \\
1
\end{array}\right] ;\left[\begin{array}{c}
1 \\
-1
\end{array}\right] ;\left[\begin{array}{c}
-1 \\
1
\end{array}\right] ;\left[\begin{array}{l}
-1 \\
-1
\end{array}\right]\right\}
$$

To apply Theorem 3, we suppose scalars $\delta, \tau_{F}$ and $\tau_{R}$ a priori fixed (as $\delta=1, \tau_{F}=1.910^{-6}$ and $\tau_{R}=1.25,10^{-8}$ ) and $M_{1}=m_{1} I$. The estimation of the stability domain is depicted in Figure 1 where 
the dotted ellipsoid represents the estimation of the region of attraction without anti-windup. By applying the numerical procedure proposed in Section 4.3, one obtains the following matrices

$$
A_{a}=\left[\begin{array}{cc}
-0.0843 & 0.0130 \\
-0.1549 & -0.1907
\end{array}\right], B_{a}=\left[\begin{array}{c}
-0.4 e^{-3} \\
0.17 e^{-3}
\end{array}\right], C_{a}=\left[\begin{array}{ll}
2.4 & 2.3
\end{array}\right], D_{a}=0.11,
$$

The associated domain of stability is in solid line in Figure 1. Note that we obtain a better estimation of the basin of attraction thanks to the anti-windup layer. Trajectories starting from several initial states are also plotted in Figure 1. They illustrate that the set $\mathcal{E}(P, \delta)$ is a good approximation of the basin of attraction of the origin. Note that some points of instability are outside of $\mathcal{E}(P, \delta)$ but very close to its boundary.

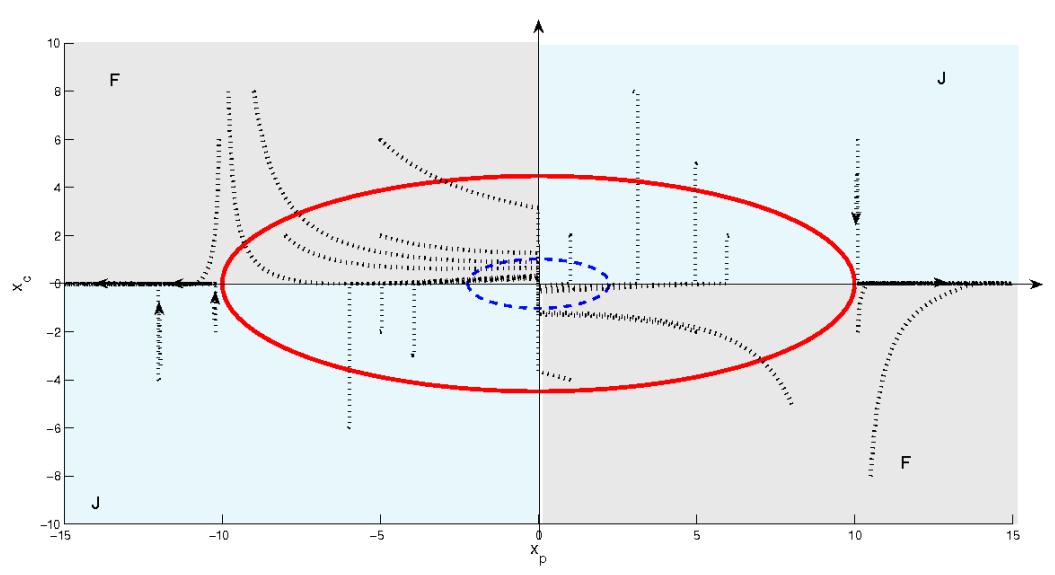

Figure 1: Region of attraction of the reset system with anti-windup (50) (solid) and without (dotted)

Considering the performance analysis, i.e., solving the optimization problem proposed in Section 4.2 subject to relation of Theorem 3, we obtain, for the same stability region, the values of $\gamma$ as follows: $\gamma=2.7$ in the linear case, $\gamma=0.8$ with reset and $\gamma=1.03$ with reset and anti-windup (see also temporal simulations in Figure 2). We emphasize that the anti-windup compensator is designed to improve performance. If the obtained $\mathcal{L}_{2}$ gain is actually better than the linear case one, the system behavior is better with reset and without anti-windup. Indeed, in this case, the anti-windup compensator acts to smooth the reset controller output $y_{c}$ to avoid saturation. As a consequence, states of the closedloop system remain in the flow set, and we do not benefit of jump action to improve output convergence. For this example anti-windup is efficient to enlarge the stability region, but not really to minimize the $\mathcal{L}_{2}$-gain.

\subsubsection{Flow and jump set case 2}

We are now applying Theorem 6 . The objective is to synthesize a new reset control rule and new flow and jump sets in order to obtain a stability domain as large as possible. We consider system (47)-(48) with the reset control law (34). Flow and jump sets satisfy (35). We solve the optimization problem proposed in Section 4.3 subject to relations of Theorem 6 to obtain sets $\mathcal{E}(P, 1)$ and $\mathcal{E}\left(P_{1}, 1\right)$ as large as possible. The anti-windup controller $(39)$ leads to $\mathcal{E}(P, 1)$, the black continuous line ellipsoid on Figure 3 . Note that this stability domain is close to the one obtained with a static anti-windup in [14]. By computing the reset law (40), we obtain a stability domain $\mathcal{E}\left(P_{1}, 1\right)$ quasi tangent to $\mathcal{E}(P, 1)$, which allows to extend the region of stability in the directions $x_{c}$. The region of stability for the closed-loop system is the union of these two sets, included in the red continuous line (the grey zone of Figure 3 ). 


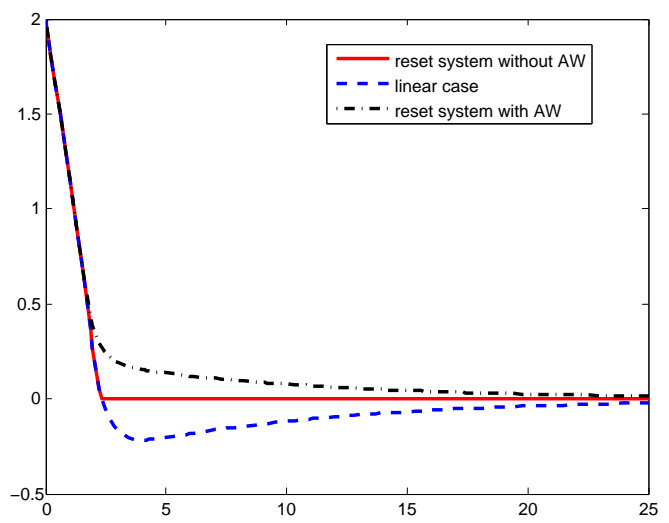

Figure 2: Output $y(t)$ of system (47)-(48) in the linear case (dashed line, $\gamma=2.7$ ), with reset (solid line $\gamma=0.8)$ and with reset and anti-windup (dotted line, $\gamma=1.03$ )

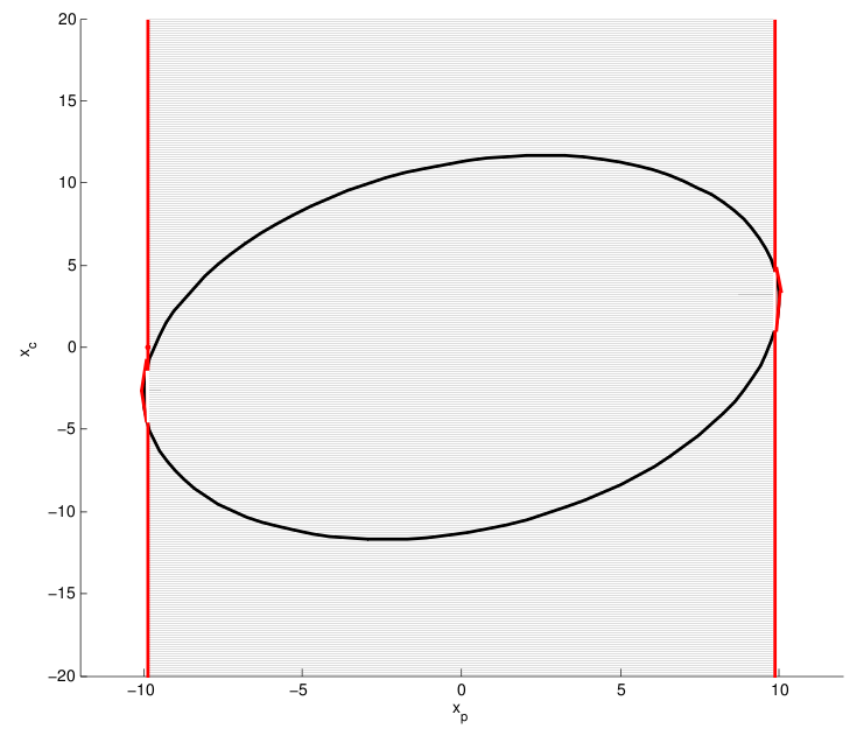

Figure 3: Stability domain of system (47)-(48) with the reset control law (33)

\section{Conclusion}

This paper dealt with the anti-windup design technique for a SISO linear system controlled by a reset controller and subject to saturation. In this context, different types of reset rule and therefore different flow and jump sets were considered. Constructive conditions leading to quasi-LMIs or LMIs, based on the use of suitable Lyapunov functions, modified sector condition and the application of adequate congruence transformations, were proposed to exhibit the matrices defining the anti-windup compensator. The computation of the anti-windup compensator aiming at ensuring both finite gain $\mathcal{L}_{2}$ stability and internal stability of the closed-loop system was then carried out from the solution of some convex optimization problems. 
A natural extension of this work could be to consider MIMO reset systems, leading to revisit the definition of reset rules, and to deal with more complex Lyapunov functions, as for example piecewise Lyapunov functions.

\section{References}

[1] O. Beker, C.V. Hollot, and Y. Chait. Plant with an integrator: an example of reset control overcoming limitations of linear feedback. IEEE Transactions on Automatic Control, 46:1797-1799, 2001.

[2] F.A. Bender, J. M. Gomes da Silva Jr., and S. Tarbouriech. A convex framework for the design of dynamic anti-windup for state-delayed systems. In American Control Conference, pages 6763-6768, Baltimore, USA, June 2010.

[3] J-M. Biannic and S. Tarbouriech. Optimization and implementation of dynamic anti-windup compensators with multiple saturations in flight control systems. Control Engineering Practice, 17:703$713,2009$.

[4] J. E. Bobrow, F. Jabbari, and K. Thai. An active truss element and control law for vibration suppression. Smart Materials and Structures, 4(4):264-269, 1995.

[5] R.T. Bupp, D.S. Bernstein, V.S. Chellaboina, and W.M. Haddad. Resetting virtual absorbers for vibration control. In American Control Conference, volume 5, pages 2647-2651, New Mexico, USA, June 1997.

[6] J.C. Clegg. A non-linear integrator for servomechanisms. Trans A.I.E.E, 77 (Part II):41-42, 1958.

[7] L. El Ghaoui, F. Oustry, and M. Ait Rami. A cone complementarity linearization algorithm for static output-feedback and related problems. IEEE Transactions on Automatic Control, 42(8):1171-1176, 1997.

[8] A. Feuer, G.C. Goodwin, and M. Salgado. Potential benefits of hybrid control for linear time invariant plants. In American Control Conference, volume 5, pages 2790-2794, New Mexico, USA, June 1997.

[9] S. Galeani, S. Tarbouriech, M.C. Turner, and L. Zaccarian. A tutorial on modern anti-windup design. European Journal of Control, 15(3-4):418-440, 2009.

[10] A.H. Glattfelder and W. Schaufelberger. Control Systems with Input and Output Constraints. Springer, 2003.

[11] R. Goebel, C. Prieur, and A. R. Teel. Smooth patchy control Lyapunov functions. Automatica, 45(3):675-683, July 2009.

[12] R. Goebel, R. G. Sanfelice, and A. R. Teel. Hybrid dynamical systems. IEEE Control Systems Magazine, 29(2):28-93, 2009 .

[13] J.M Gomes da Silva Jr. and S. Tarbouriech. Local stabilization of discrete-time linear systems with saturating controls : an LMI-based approach. IEEE Transactions on Automatic Control, 46(1):119$124,2001$.

[14] J.M Gomes da Silva Jr. and S. Tarbouriech. Anti-windup design with guaranteed regions of stability: an LMI-based approach. IEEE Transactions on Automatic Control, 50(1):106-111, 2005. 
[15] G. Grimm, J. Hatfield, I. Postlethwaite, A.R. Teel, M.C. Turner, and L. Zaccarian. Antiwindup for stable linear systems with input saturation: An LMI-based synthesis. IEEE Transactions on Automatic Control, 48(9):1509-1525, 2003.

[16] W. M. Haddad, V.S. Chellaboina, and N. A. Kablar. Active control of combustion instabilities via hybrid resetting controllers. In American Control Conference, pages 2378-2382, June 2000.

[17] T. Hu and Z. Lin. Control Systems with Actuator Saturation: Analysis and Design. Boston, MA: Birkhäuser, 2001.

[18] T. Hu, A.R. Teel, and L. Zaccarian. Anti-windup synthesis for linear control systems with input saturation: Achieving regional, nonlinear performance. Automatica, 44(2):515-519, 2008.

[19] M.L. Kerr, M.C. Turner, and I. Postlethwaite. Practical approaches to low-order anti-windup compensator design: a flight control comparison. In World IFAC Congress, Seoul, Korea, 2008.

[20] T. Loquen, S. Tarbouriech, and C. Prieur. Stability analysis for reset systems with input saturation. In Conference on Decision and Control, pages 3272-3277, New Orleans, LA, USA, 2007.

[21] D. Nešic̀, L. Zaccarian, and A.R. Teel. Stability properties of reset systems. Automatica, 44(8):20192026, 2008.

[22] C. Prieur, R. Goebel, and A.R. Teel. Hybrid feedback control and robust stabilization of nonlinear systems. IEEE Transactions on Automatic Control, 52(11):2103-2117, 2007.

[23] C. Prieur, S. Tarbouriech, and L. Zaccarian. Guaranteed stability for nonlinear systems by means of a hybrid loop. In IFAC Symposium on Nonlinear Control Systems (NOLCOS), pages 72-77, Bologna, Italy, September 2010.

[24] C. Prieur and A. R. Teel. Uniting local and global output feedback controllers. IEEE Transactions on Automatic Control, to appear, 2011.

[25] C. Roos and J-M. Biannic. A convex characterization of dynamically-constrained anti-windup controllers. Automatica, 44(9):2449-2452, 2008.

[26] C. Scherer, P. Gahinet, and M. Chilali. Multi-objective output-feedback control via LMI optimization. IEEE Transactions on Automatic Control, 42(7):896-911, 1997.

[27] S. Tarbouriech, G. Garcia, and A.H. Glattfelder (Eds.). Advanced strategies in control systems with input and output constraints, volume 346 of LNCIS. Springer Verlag, 2007.

[28] S. Tarbouriech, C. Prieur, and J.M. Gomes da Silva Jr. Stability analysis and stabilization of systems presenting nested saturations. IEEE Transactions on Automatic Control, 51(8):1364-1371, 2006.

[29] S. Tarbouriech and M.C. Turner. Anti-windup design: an overview of some recent advances and open problems. IET Control Theory and Application, 3(1):1-19, 2009.

[30] A.R. Teel and N. Kapoor. The $\mathcal{L}_{2}$ anti-windup problem: Its definition and solution. In European Control Conference, Brussels, Belgium, july 1997.

[31] M.C. Turner and I. Postlethwaite. A new perspective on static and low order anti-windup synthesis. International Journal of Control, 77(1):27-44, 2004.

[32] L. Zaccarian, D. Nešic̀, and A.R. Teel. First order reset elements and the Clegg integrator revisited. In American Control Conference, pages 563-568, Portland, OR, USA, June 2005. 
[33] L. Zaccarian and A.R. Teel. A common framework for anti-windup, bumpless transfer and reliable designs. Automatica, 38(10):1735-1744, 2002.

[34] Y. Zheng, Y. Chait, C.V. Hollot, M. Steinbuch, and M. Norg. Experimental demonstration of reset control design. Control Engineering Practice, 8(2):113-120, 2000. 